\title{
Sulforaphane has a therapeutic effect in an atopic dermatitis murine model and activates the Nrf2/HO-1 axis
}

\author{
WENQING WU ${ }^{1}$, GE PENG $^{2}$, FAN YANG $^{1}$, YUE ZHANG $^{1}$, ZHENZHEN MU $^{1}$ and XIUPING HAN ${ }^{1}$ \\ ${ }^{1}$ Department of Dermatology, Shengjing Hospital of China Medical University, Shenyang, \\ Liaoning 110004, P.R. China; ${ }^{2}$ Atopy (Allergy) Research Center, Department of Dermatology and Allergology, \\ Juntendo University Graduate School of Medicine, Tokyo 1138642, Japan
}

Received October 25, 2018; Accepted May 14, 2019

DOI: $10.3892 / \mathrm{mmr} .2019 .10405$

\begin{abstract}
Atopic dermatitis (AD), a chronic inflammatory skin disease, is characterized by intense itching and recurrent eczematous lesions. Sulforaphane is known to attenuate oxidative stress, and tissue or cell damage in cerebral ischemia, brain inflammation and intracerebral hemorrhage. In the present study, a 2,4-dinitrochlorobenzene (DNCB)-induced AD mouse model was developed, and ear thickness, dermatitis score, eosinophil count, mast cell infiltration, and serum IgE levels were measured in DNCB-induced AD and sulforaphane-treated groups to demonstrate the therapeutic effects of sulforaphane. AD symptoms of DNCB-induced mice were attenuated by sulforaphane treatment compared with those of negative control mice; furthermore, eosinophil count, mast cell infiltration and serum IgE levels were also reduced by sulforaphane treatment in DNCB-induced AD mice. Western blot assays revealed that the expression levels of nuclear factor-E2-related factor 2 (Nrf2) and heme oxygenase-1 (HO-1), which exhibit oxidation resistance, were increased by sulforaphane treatment in DNCB-induced AD mice. The present study suggested that sulforaphane exerted a therapeutic effect in the AD mouse model through the activation of the Nrf2/HO-1 axis as well as the suppression of Janus kinase 1/STAT3 signaling pathway.
\end{abstract}

\section{Introduction}

Atopic dermatitis (AD) is a chronic inflammatory disease of skin that is characterized by intense itching and recurrent eczematous lesions (1). The main pathological changes associated with $\mathrm{AD}$ are immunologic disturbance and skin barrier disorders (2). Topical use of glucocorticoids or

Correspondence to: Dr Xiuping Han, Department of Dermatology, Shengjing Hospital of China Medical University, 36 Sanhao Street, Heping, Shenyang, Liaoning 110004, P.R. China

E-mail: drhanxiuping@sina.com

Key words: sulforaphane, atopic dermatitis, 2,4-dinitrochlorobenzene, nuclear factor-E2-related factor 2/heme oxygenase 1 axis, Janus kinase 1/STAT3 calcineurin inhibitors are the predominant methods for the management of $\mathrm{AD}$, and systemic anti-inflammatory treatment with glucocorticosteroids (short term), cyclosporine (in adults) or azathioprine is used in some severe AD cases (3). Considering the important role of anti-inflammatory therapy in the treatment of dermatitis, the present study sought to evaluate the effectiveness of novel anti-inflammatory agents in the treatment of AD.

Previous studies have demonstrated that highly adverse conditions lead to the generation of oxidative stress in skin tissue and that this phenomenon can stimulate the occurrence of $\mathrm{AD}(4,5)$. The transcription factor nuclear factor-E2-related factor 2 (Nrf2), which is involved in phase II detoxification, inflammatory signaling, DNA repair and antioxidant responses in cellular defense, is considered as a protective factor against oxidative modification in keratinocytes, melanocytes and fibroblasts, and improves skin barrier function and photoprotection $(4,6)$. Nrf2-deficient skin fibroblasts are more susceptible to increased inflammation than normal skin fibroblasts in the skin under UVA irradiation (7). Sulforaphane is a natural dietary isothiocyanate extracted from cruciferous vegetables and it can increase the antioxidative ability in tissues following focal cerebral ischemia, brain inflammation, intracerebral hemorrhage and many types of inflammation (8). A recent study demonstrated that sulforaphane can stimulate the expression of $\mathrm{Nrf} 2$ in human skin fibroblasts and decrease DNA double-strand breaks after exposure to ionizing radiation; these results suggest that sulforaphane could protect the skin from ionizing radiation-induced injury by upregulating the expression of $\mathrm{Nrf} 2$ (9). In addition, an antioxidant gene, heme oxygenase-1 (HO-1), whose expression is induced by increased Nrf2 expression, has been shown to be an anti-inflammatory factor that protects the skin tissue against oxidative stress (10).

The high expression of serum IgE in response to exogenous and endogenous allergens in patients with $\mathrm{AD}$ is associated with severe skin inflammation, and chronicity of AD and indicates poor long-term prognosis for patients (11-13). To illustrate the role $\operatorname{IgE}$ in $\mathrm{AD}$, serum $\operatorname{IgE}$ autoantibodies have been identified by immunostaining, which indicated that $\operatorname{IgE}$ is expressed at a high level in the keratinocytes of patients with AD (14). In a clinical study, anti-IgE therapy demonstrated positive effects in controlling the development of AD (15). Eosinophils and mast cells also have an important role in the 
prognosis of AD and there is some evidence that eosinophils can not only activate the proinflammatory process but also participate in tissue repair and the fibrotic processes of allergic inflammation (16). Chemokines produced by mast cells can also promote the development of $\mathrm{AD}$, as demonstrated by treatment of mast cells with dexamethasone and a calcineurin inhibitor (FK506) (17).

In the present study, sulforaphane reduced epithelial thickness, serum IgE level and infiltration of eosinophils and mast cells in AD epithelial tissue and increased the levels of Nrf2, phosphorylated (p-)Nrf2 and HO-1 and reduced the levels of p-Janus kinase 1 (JAK1) and p-STAT3. This indicated that sulforaphane can reduce the level of inflammation in the skin of AD mice model and it may have a curative effect on patients with AD.

\section{Materials and methods}

Animals. A total of 40 Female BALB/c mice, aged 6 weeks, were purchased from Beijing HFK Bioscience Co., Ltd. They were housed under specific pathogen-free conditions at a controlled temperature of $20-25^{\circ} \mathrm{C}$ and $35-75 \%$ humidity with a 12-h light/dark cycle. The animals were provided with sterile food and water ad libitum. All animal care and experiments were performed in the Experimental Animal Center in accordance with the national guidelines and were approved by the animal care committee of Shengjing Hospital of China Medical University (approval no. 2016PS001K).

Induction of $A D$-like lesions and sulforaphane administration. After a week of acclimation, the mice were divided into 5 groups ( $n=8$ per group): i) Vehicle, vehicle + phosphate buffer saline (PBS); ii) AD, dinitrochlorobenzene (DNCB) + PBS; iii) SFN2.5, DNCB + sulforaphane intraperitoneal (i.p.) injection $(2.5 \mathrm{mg} / \mathrm{kg})$; iv) SFN5, DNCB + sulforaphane i.p. injection $(5 \mathrm{mg} / \mathrm{kg})$; and v) SFN10, DNCB + sulforaphane i.p. injection $(10 \mathrm{mg} / \mathrm{kg})$. The sulforaphane doses were selected and modified according to previous studies (18-20).

The dorsal hair of mice was completely removed with an electric razor before the day of administration (an area of $\sim 4 \mathrm{~cm}^{2}$ ). On the first day, $150 \mu 1$ 1\% DNCB (Sigma-Aldrich; Merck KGaA) dissolved in a mixture containing acetone and olive oil $(3: 1 \mathrm{v} / \mathrm{v})$, was dropped on the dorsal skin, and $20 \mu \mathrm{l}$ $1 \%$ DNCB solution was dropped in ears. On the 5th day, 150 or $20 \mu 10.2 \%$ DNCB solution dissolved in an acetone and olive oil mixture $(3: 1 \mathrm{v} / \mathrm{v})$, respectively, were applied to the dorsal skin and ears three times a week for 3 weeks (days 5-23). For the vehicle group, the same dose of the mixture comprising acetone and olive oil (3:1 v/v) was applied to the dorsal skin and ears of the mice.

Then, $1 \mathrm{~h}$ after each DNCB application, sulforaphane at the doses of $2.5,5$ and $10 \mathrm{mg} / \mathrm{kg}$ i.p. or PBS i.p. was injected (days 1-23), for a total of 10 times. When the experiments were completed, the animals were anesthetized with $2 \%$ isoflurane, and blood, dorsal dermal tissue and ear tissue were collected for analysis. The experimental schedule is summarized in Fig. 1.

Evaluation of severity of dermatitis. Dermatitis in each mouse was observed and the score was recorded once a week according to the criteria described previously (21). The severity of dermatitis was assessed according to four symptoms: i) Erythema/hemorrhage; ii) scar/dryness; iii) edema; and iv) excoriation/erosion. The score of each clinical symptom ranged from 0 to 3 (none, 0 ; mild, 1 ; moderate, 2 ; and severe, 3 ). The total dermatitis score (maximum score 12) was the sum of individual scores. Additionally, ear thickness of the mice was measured and recorded once a week by using a micrometer (Mitutoyo Kawasaki). On day 24, the mice were anesthetized with $2 \%$ isoflurane before sacrifice and the dorsal lesions were imaged using a digital camera (Praktica Luxmedia16-Z21C; Pentacon $\mathrm{GmbH}$ ).

Evaluation of scratching behavior and ear thickness. To avoid statistical bias between groups, the number of scratches in each mouse was recorded before administration. Mice with high and low number of scratches were excluded from the experiment, and the remaining mice were randomly divided into five groups. Each mouse was observed for $10 \mathrm{~min}$, and the number of scratches was recorded and videotaped once a week. A scratching event was defined as the mouse rubbing the dorsal skin and ears with the hind paws. Moreover, when the continuous scratch time exceeded $3 \mathrm{sec}$, it was recorded as two scratches and the scratching was terminated by human intervention. In addition, mouse ear thickness was measured and recorded once a week by using a micrometer (Mitutoyo Kawasaki). All data measurements were performed by a single investigator to avoid inter-observer variation.

Histological analysis. To assess epidermal thickness and inflammatory cell infiltration (i.e., eosinophils and mast cells), the dorsal skin lesions of the mice were fixed in $10 \%$ paraformaldehyde for $24 \mathrm{~h}$ at $37^{\circ} \mathrm{C}$ on the last day, embedded in paraffin and $4-\mu \mathrm{m}$-thick paraffin sections were made. Hematoxylin and eosin (H\&E) staining and toluidine blue staining were then performed for $30 \mathrm{sec}$ each at $37^{\circ} \mathrm{C}$ to identify epidermal thickness and inflammatory cells of each group, respectively. The number of eosinophils and mast cells in each section was obtained from five random views under x400 magnification. Tissue sections were observed using an inverted microscope (Y-TV55; Nikon Corporation) and the data were obtained from five sections per mouse. Histopathological evaluation of all skin sections was carried out in a blind manner.

Serum IgE measurements. Abdominal aortic blood of mice was collected on day 24. IgE levels were measured with an ELISA kit (cat. no. E01G0277; Abcam) in accordance with the manufacturer's instructions.

cDNA synthesis and reverse transcription-quantitative PCR $(R T-q P C R)$. Total RNA of mouse dorsal skin in each group was extracted with TRIzol reagent (Invitrogen; Thermo Fisher Scientific, Inc.). RNA extraction was performed according to the manufacturer's protocol. cDNA was prepared from $1 \mu \mathrm{g}$ RNA using RevertAid Reverse Transcriptase (Thermo Fisher Scientific, Inc.) and incubated for $4 \mathrm{~h}$ at $37^{\circ} \mathrm{C}$ and $5 \% \mathrm{CO}_{2}$. RT-qPCR analyses were performed on the 7500 Real-Time PCR System (Applied Biosystems; Thermo Fisher Scientific, Inc.). Cycling conditions were $3 \mathrm{~min}$ at $95^{\circ} \mathrm{C}$ 


\section{Schedule of experiment}

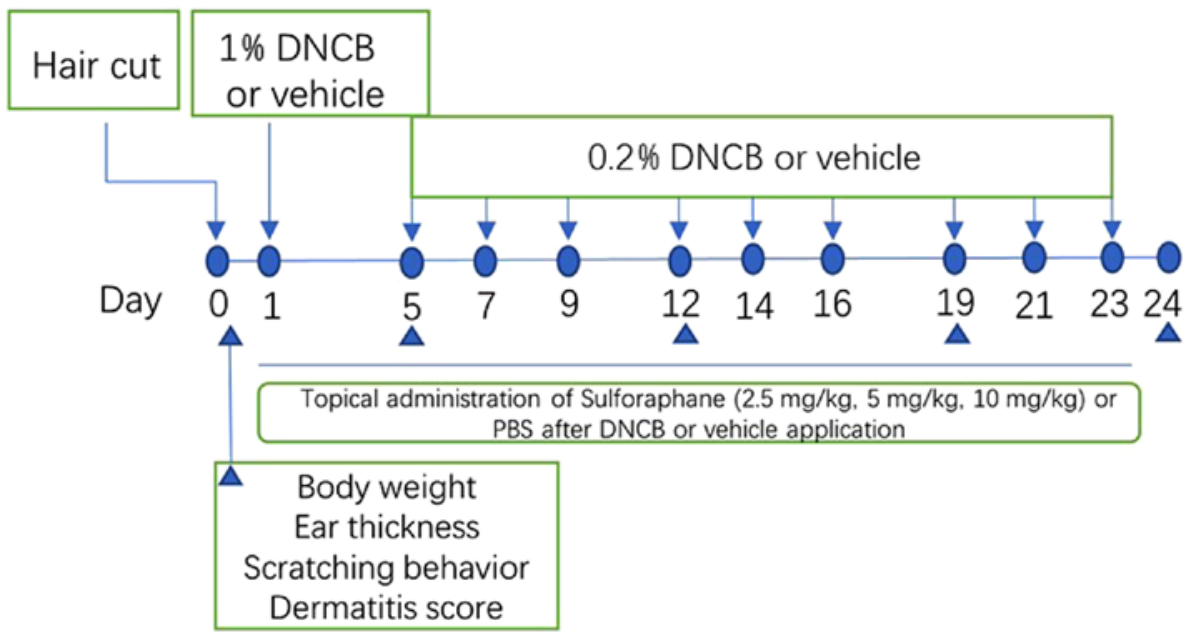

Figure 1. Experimental schedule. To induce atopic dermatitis, DNCB was topically applied to BALB/c mice. The dorsal hair was completely removed the day before the application. On the first day, $1 \%$ DNCB or vehicle was administered. From the fifth day onwards, $0.2 \%$ DNCB or vehicle was administered three times a week for 3 weeks (days 5-24), and $1 \mathrm{~h}$ later, sulforaphane 2.5, 5 and $10 \mathrm{mg} / \mathrm{kg}$ or PBS was separately i.p. injected. DNCB, 2,4-dinitrochlorobenzene.

followed by 60 cycles of $30 \mathrm{sec}$ at $95^{\circ} \mathrm{C}, 30 \mathrm{sec}$ at $56^{\circ} \mathrm{C}$ and $30 \mathrm{sec}$ at $72^{\circ} \mathrm{C}$. The expression level of genes was detected using the following primers: Interleukin (IL)-6, 5'-TTG CCTTCTTGGGAC-3' (forward), 5'-TTGCCATTGCAC AACTCTT-3' (reverse); IL-1 $\beta$, 5'-CTGTCGGACCCATATG AGC-3' (forward), 5'-GCTCATGGAGAATATCACTTG TTG-3' (reverse); tumor necrosis factor- $\alpha$ (TNF- $\alpha), 5^{\prime}-\mathrm{AAG}$ CCTGTAGCCCACGTCGTA-3' (forward), 5'-GGCACCACT AGTTGGTTGTCTTTG-3' (reverse); mouse GAPDH, 5'-AAA TGGTGAAGGTCGGTGTG-3' (forward), 5'-TGAAGGGGT CGTTGATGG-3' (reverse). All experiments were performed in duplicate. For relative quantification analyses, a comparative $2^{-\Delta \Delta \mathrm{Cq}}$ method was used (22), where the median value of the vehicle group was used as the calibrator.

Western blot analysis. Western blotting was used to detect the protein levels of Nrf2, p-Nrf2, HO-1, p-JAK1, JAK1, p-STAT3 and STAT3. Dorsal skin tissues were processed by the protein extraction kit and each sample in one group was homogenized in RIPA buffer (BioLegend, Inc.) and then centrifuged for $30 \mathrm{~min}$ at $12,000 \mathrm{x} \mathrm{g}$ and $4^{\circ} \mathrm{C}$ to collect the supernatant. Then, eight protein samples from each group were pooled together, separated by SDS-PAGE and then transferred onto a PVDF membrane (EMD Millipore). The quantification of protein concentration was performed by using bichinchoninic acid protein assay kit (Pierce, Thermo Fisher Scientific, Inc.): $20 \mu \mathrm{g}$ protein from each sample was separated by SDS-PAGE on a $10 \%$ gel and transferred onto a polyvinylidene difluoride membrane and then blocked with $5 \%$ non-fat milk for $2 \mathrm{~h}$ at $37^{\circ} \mathrm{C}$. The membrane was incubated with monoclonal rabbit anti-mouse Nrf2 (cat. no. 2772, 1:10,000 dilution), p-Nrf2 (cat. no. 9524, 1:10,000 dilution), HO-1 (cat. no. 2882, 1:10,000 dilution), p-JAK1 (cat. no. 4970, 1:10,000 dilution), JAK1 (cat. no. 8675, 1:10,000 dilution), p-STAT3 (cat. no. 4462 , 1:10,000 dilution), STAT3 (cat. no. 6643, 1:10,000 dilution) and polyclonal rabbit anti-mouse HO-1 (cat. no. 4480, 1:10,000 dilution, all from Abcam), respectively, along with monoclonal rabbit anti-mouse tubulin (cat. no. SC-2357, 1:10,000 dilution; ProteinTech Group, Inc.) as an internal reference for $15 \mathrm{~h}$ at $4^{\circ} \mathrm{C}$, followed by incubation with Alexa Fluor 800-labeled goat anti-rabbit IgG (Invitrogen; Thermo Fisher Scientific, Inc.) for $1.5 \mathrm{~h}$ at $37^{\circ} \mathrm{C}$. Images were acquired using an AI600 (BD Biosciences). Band intensities (pixels $/ \mathrm{mm}^{2}$ ) were obtained using Image Quant 5.2 software (Molecular Dynamics) after subtracting the background intensities. The values of the sulforaphane-treated group values were normalized to those of $\beta$-actin as normal control (NC).

Statistical analysis. GraphPad Prism software 7.0 (GraphPad Software, Inc.) was used to analyze the statistical data, and the data were expressed as mean \pm SEM. For comparison of multiple groups data, one-way or two-way ANOVA followed by Dunnett's post hoc test or Tukey's honest significant difference (HSD) test to detect the differences in these groups. All the experiments were performed in triplicate. $\mathrm{P}<0.05$ was considered to indicate a statistically significant difference.

\section{Results}

Sulforaphane has a protective effect against DNCB-induced $A D$ in mice. To study the therapeutic effects of sulforaphane on skin lesions in AD mice, AD mice were treated with a concentration gradient of sulforaphane $(2.5,5$ and $10 \mathrm{mg} / \mathrm{kg})$, with the different groups termed SFN2.5, SFN5 and SFN10, respectively. The AD model group exhibited severe dermatitis with erythema, scarring, edema and erosion (Fig. 2A). The dermatitis scores gradually increased throughout the 3-week experimental period (Fig. 2B). Skin condition was significantly improved in sulforaphane-treated groups compared with those in the AD group (Fig. 2A and B). Moreover, sulforaphane decreased the dermatitis score of DNCB-induced skin lesions on days 14 and 21 in a dose-dependent manner. 


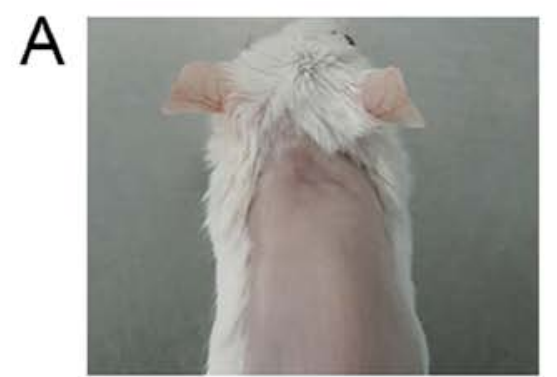

Vehicle

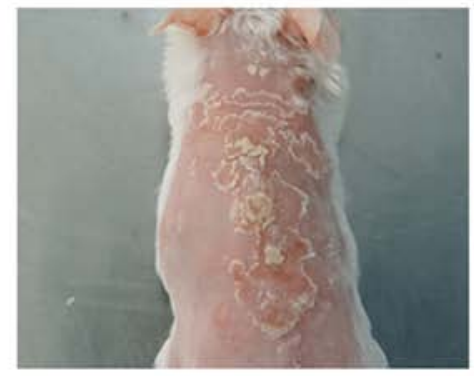

AD+SFN5

B
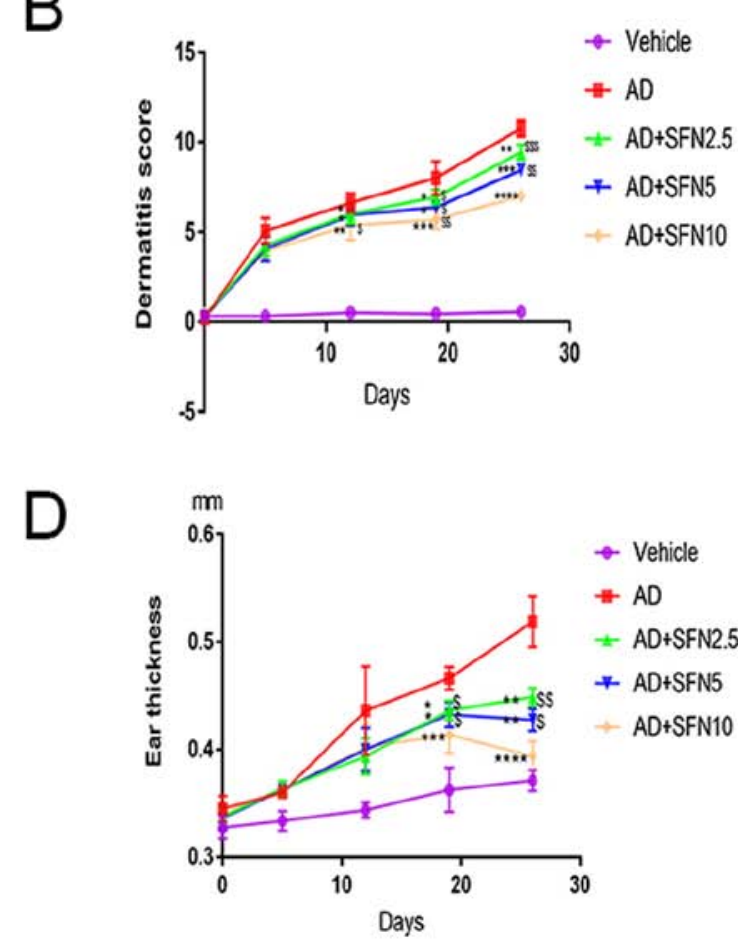

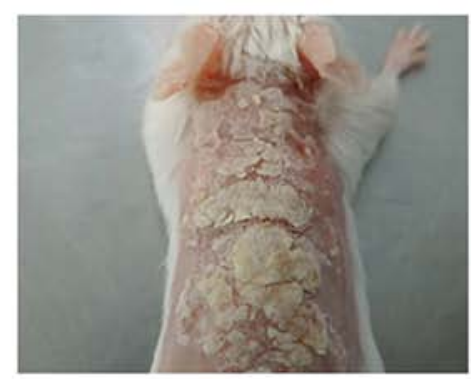

$\mathrm{AD}$

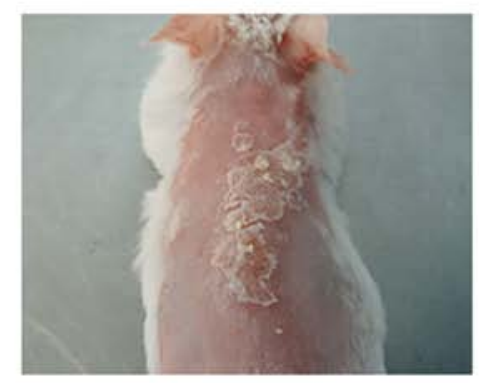

$\mathrm{AD}+\mathrm{SFN} 10$

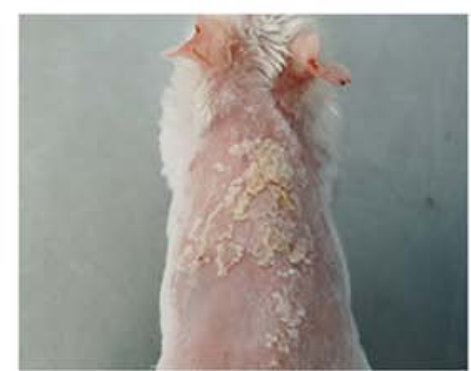

AD+SFN2.5
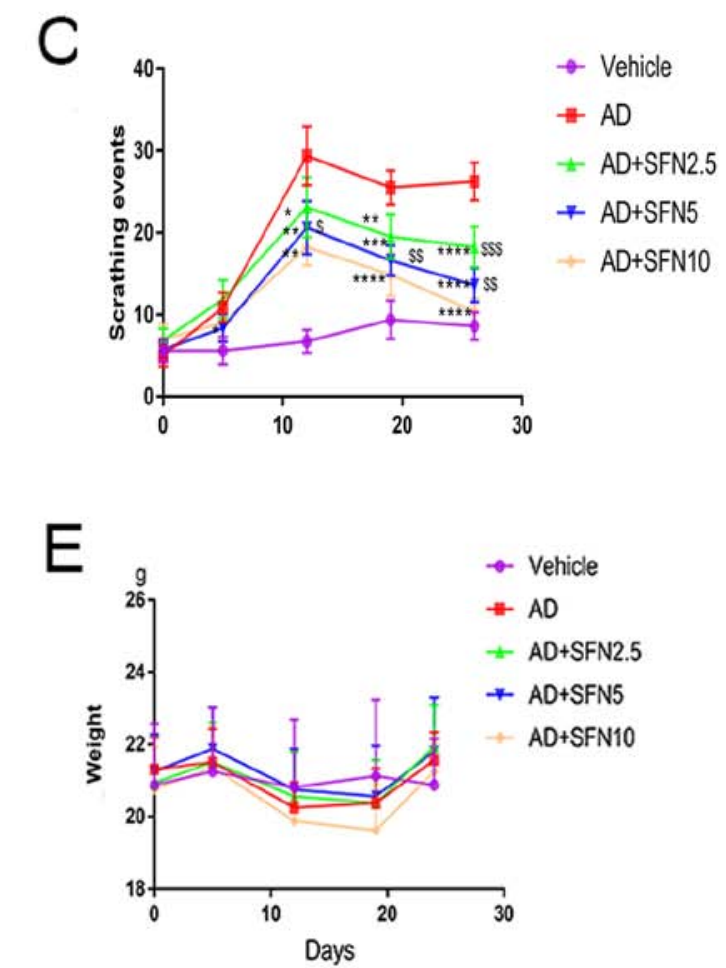

Figure 2. Effects of SFN on atopic dermatitis-like symptoms in BALB/c mice. (A) Images of skin lesions from the groups were taken on the last day of the experiment (day 24). (B) Dermatitis scores were evaluated once a week for 5 weeks (two-way ANOVA analysis followed by Dunnett's post hoc test). (C) The number of scratching events was recorded for 10 min for each mouse in a cage once a week (two-way ANOVA analysis followed by Dunnett's post hoc test). (D) Ear thickness was measured once a week for 5 weeks using a micrometer (two-way ANOVA analysis followed by Dunnett's post hoc test). (E) Mouse body weight was recorded once a week (two-way ANOVA followed by Tukey's honest significant difference test). Results are expressed as the mean \pm SEM $(n=8) .{ }^{*} \mathrm{P}<0.05,{ }^{* *} \mathrm{P}<0.01,{ }^{* * *} \mathrm{P}<0.001$ and ${ }^{* * * * *} \mathrm{P}<0.0001$ vs. AD group. ${ }^{\$} \mathrm{P}<0.05,{ }^{\$} \mathrm{P}<0.01$ and ${ }^{\$ \$ \$} \mathrm{P}<0.001$ vs. AD $+\mathrm{SFN} 10$ group. DNCB, $2,4-$ dinitrochlorobenzene; SFN, sulforaphane; Vehicle, vehicle + PBS-treated group; AD, DNCB + PBS-treated atopic dermatitis group; AD + SFN2.5, DNCB + SFN (2.5 mg/kg i.p.)-treated group; AD + SFN5, DNCB + SFN (5 mg/kg i.p.)-treated group; AD + SFN10, DNCB + SFN (10 mg/kg i.p.)-treated group.

Scratching behavior was the most noticeable clinical feature of AD (Fig. 2A and B).

The results of monitoring mouse scratching behavior showed that scratching events increased rapidly in the DNCB-induced AD group and were maintained at a high level during the 3-week experimental period compared to those of the vehicle group (Fig. 2C). However, after treatment with sulforaphane, the number of scratching events was rapidly decreased in the SFN2.5, SFN5, and SFN10 groups compared with those of the AD group (Fig. 2C). Moreover, the SFN10 

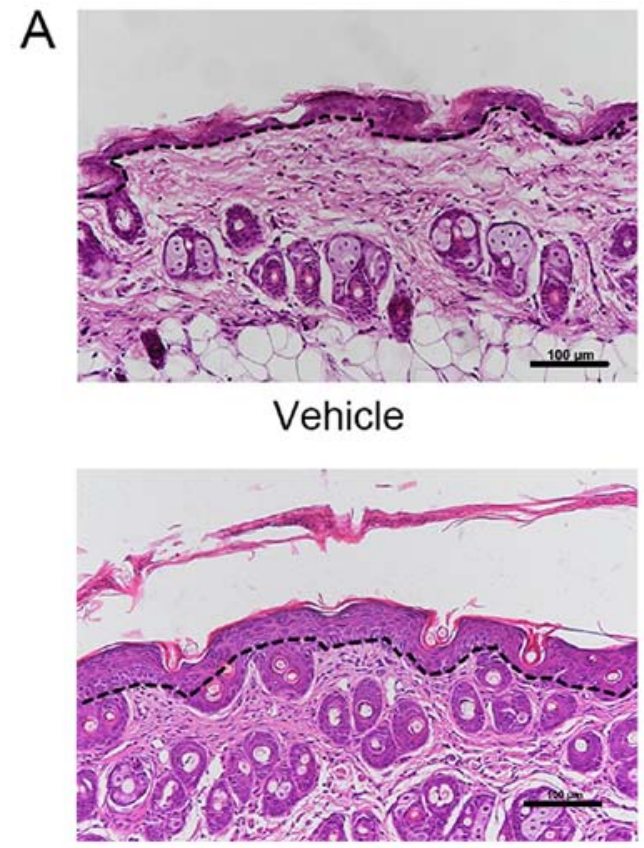

$\mathrm{AD}+\mathrm{SFN} 2.5$

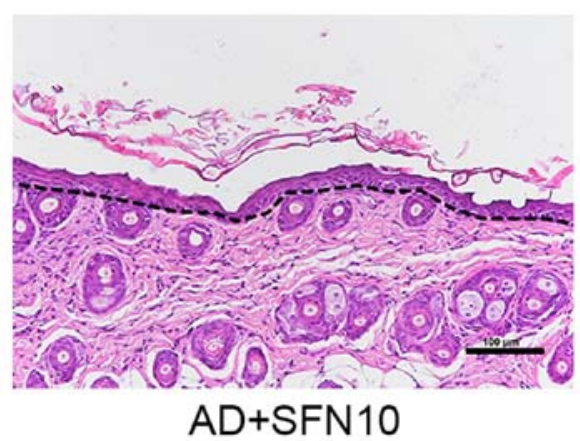

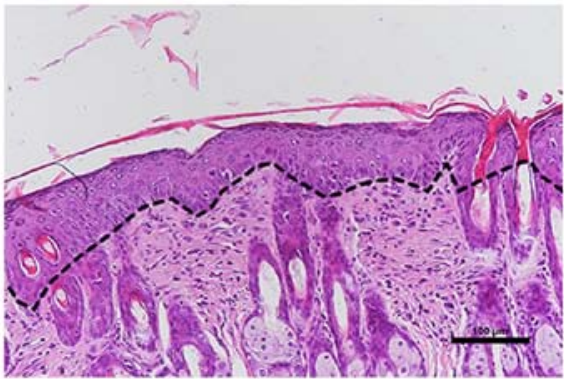

$\mathrm{AD}$

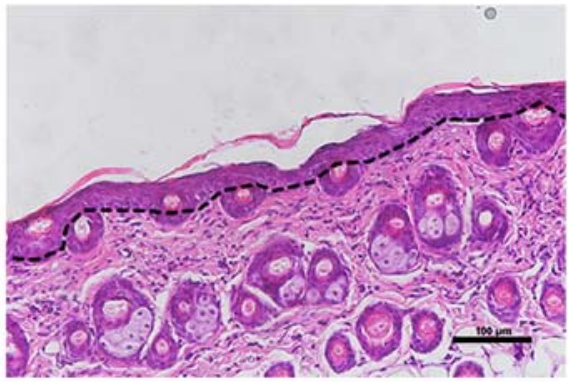

AD+SFN5

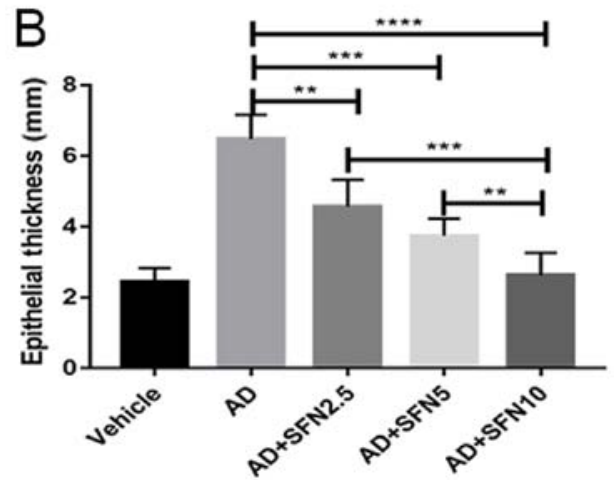

Figure 3. Effects of SFN on dorsal skin thickness in atopic dermatitis mouse skin lesions (5 fields per animal). (A) Epidermal thickness was presented in hematoxylin and eosin-stained sections (x20; scale bar, $100 \mu \mathrm{m}$ ). The black dotted line indicates the boundary line between the epidermis and the dermis. (B) Measurement of epidermal thickness. Results are expressed as the mean \pm SEM $(n=8) .{ }^{* *} \mathrm{P}<0.01,{ }^{* * *} \mathrm{P}<0.001$ and ${ }^{* * * *} \mathrm{P}<0.0001$. One-way ANOVA analysis followed by Dunnett's post hoc test. DNCB, 2,4-dinitrochlorobenzene; SFN, sulforaphane; Vehicle, vehicle + PBS-treated group; AD, DNCB + PBS-treated atopic dermatitis group; AD + SFN2.5, DNCB + SFN (2.5 mg/kg i.p.)-treated group; AD + SFN5, DNCB + SFN (5 mg/kg i.p.)-treated group; AD + SFN10, $\mathrm{DNCB}+\mathrm{SFN}(10 \mathrm{mg} / \mathrm{kg}$ i.p.)-treated group.

group had lower scratching events than the SFN2.5 group $(\mathrm{P}<0.01)$ and the SFN5 group $(\mathrm{P}<0.001$; Fig. 2C). Further, SFN2.5, SFN5 and SFN10 groups also exhibited a significant reduction in DNCB-induced ear thickness compared to that of the AD group (two-way ANOVA analysis followed by Dunnett's post hoc test; Fig. 2D). To determine the toxicity of sulforaphane, changes in weight of each mouse were recorded and found that sulforaphane had no effect on the maintenance of body weight (two-way ANOVA analysis followed by Tukey's HSD test; Fig. 2E) that there was a significant difference in changes weight by different concentration SFN treatment.

Previous studies have indicated that DNCB-induced AD-like lesions have high levels of inflammatory cell infiltration, leading to thickening of the skin (23). H\&E staining revealed that the epidermal thickness of the dorsal skin was attenuated by treatment in the SFN2.5 SFN5, and SFN10 groups compared with the AD group (one-way ANOVA analysis followed by Dunnett's post hoc test). The epidermal thickness was significantly lower in the SFN10 group compared with the SFN2.5 and SFN5 groups ( $\mathrm{P}<0.01$; Fig. $3 \mathrm{~A}$ and $\mathrm{B})$.

Sulforaphane reduced eosinophil accumulation, mast cell infiltration, and serum IgE levels in AD mice. Eosinophil levels are elevated in most AD patients and are closely associated with disease activity. In this study, H\&E staining of the drug-administered dorsal skin was performed and eosinophil infiltration was observed under an optical microscope for each group. The number of eosinophils in skin was reduced in SFN2.5, SFN5, and SFN10 groups compared with the number in the AD group (one-way ANOVA analysis followed by Dunnett's post hoc test; Fig. 4).

Increased serum IgE levels and mast cell infiltration in skin tissue are the main features of AD (24). Toluidine blue staining of the dorsal skin lesions was performed and the infiltration of mast cells in the dermis was observed under the optical microscope (Fig. 5A). A large number of mast cells appeared in the dermis of the AD group. The number of mast 

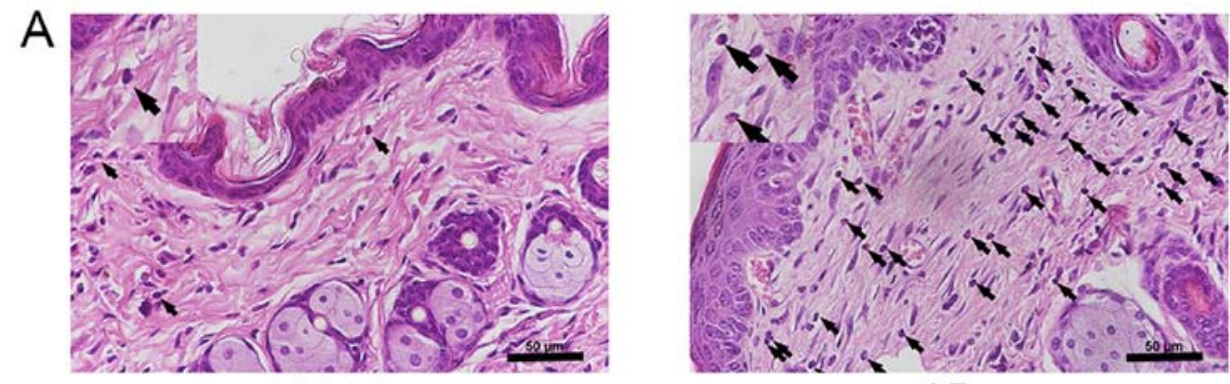

Vehicle

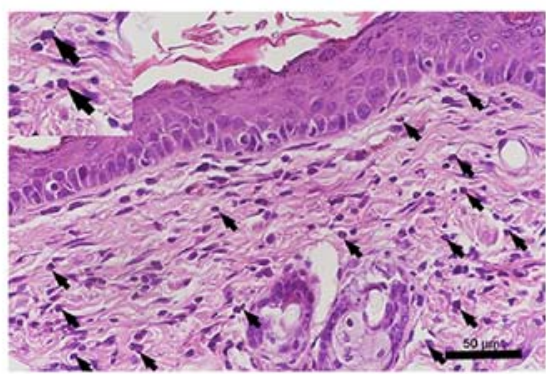

$A D+S F N 2.5$

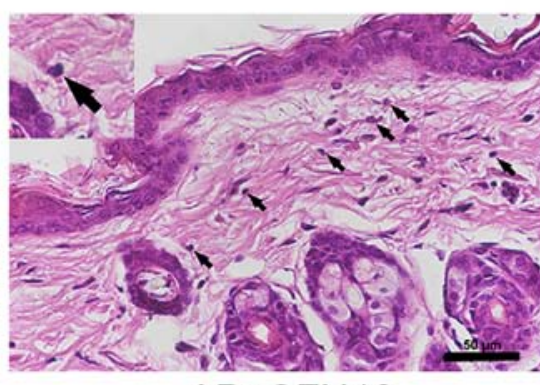

$A D+S F N 10$

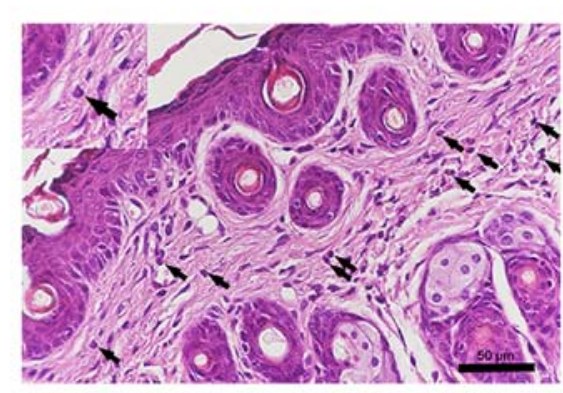

$A D+S F N 5$

$B$

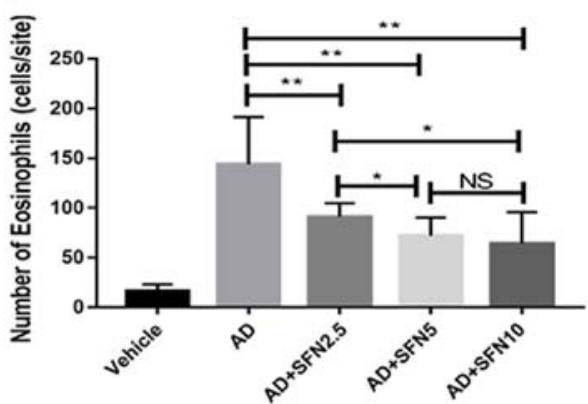

Figure 4. Effects of SFN on eosinophil accumulation in atopic dermatitis mouse skin lesions (5 fields per animal). (A) Eosinophils were presented in hematoxylin and eosin-stained sections (x40; scale bar, $50 \mu \mathrm{m}$ ), and the morphology of eosinophils was clearly observed in the partially enlarged image on the left upper corner. Black arrows indicate stained eosinophils. (B) Average number of eosinophils in five sites chosen randomly was counted at x400 magnification. The enlargement part of each groups was enlarged by 2.5 times. Results are expressed as the mean \pm SEM $(n=8)$. ${ }^{*} \mathrm{P}<0.05$ and ${ }^{* *} \mathrm{P}<0.01$. One-way ANOVA analysis followed by Dunnett's post hoc test. DNCB, 2,4-dinitrochlorobenzene; SFN, sulforaphane; Vehicle, vehicle + PBS-treated group; AD, DNCB + PBS-treated group; AD + SFN2.5, DNCB + SFN (2.5 mg/kg i.p.)-treated group; AD + SFN5, DNCB + SFN (5 mg/kg i.p.)-treated group; AD + SFN10, DNCB + SFN $(10 \mathrm{mg} / \mathrm{kg}$ i.p.)-treated group; NS, no significance.

cells decreased significantly after sulforaphane treatment, and the effect was significantly increased with the increasing dose of in the SFN2.5, SFN5 and SFN10 groups compared with the AD group (one-way ANOVA analysis followed by Dunnett's post hoc test; Fig. 5B). The effect of sulforaphane on total serum IgE levels was also investigated. The results showed that serum IgE levels were significantly elevated in the AD group; however, increased serum IgE levels were significantly attenuated in the SFN2.5, SFN5 and SFN10 groups compared with those in the AD group, while the SFN10 group had lower levels of serum IgE than the SFN2.5 group $(\mathrm{P}<0.05)$ and the SFN5 group $(\mathrm{P}<0.01$; Fig. $6 \mathrm{~A})$.

Sulforaphane increases the expression of $\mathrm{Nrf} 2$ and $\mathrm{HO}-1$, and downregulated the JAK2/STAT3 pathway and the levels of $I L-6, I L-1 \beta$ and $T N F-\alpha$ in $A D$ mice. To explicate antioxidant and anti-inflammatory effects of sulforaphane in AD mice, western blot assays were performed to detect the expression of Nrf2, p-Nrf2, HO-1, p-JAK1, JAK1, p-STAT3 and STAT3 in full-thickness dorsal skin. The results showed that p-Nrf2,Nrf2 and HO-1 were slightly elevated in the AD group compared with that in the vehicle group. However, the levels of p-Nrf2, Nrf, and HO-1 in the SFN2.5, SFN5, and SFN10 groups were significantly higher than those in the AD group; furthermore, the SFN10 group exhibited higher levels of Nrf2 and HO-1 expression than the SFN2.5 and SFN5 groups (Fig. 6B). By contrast, the mRNA expression levels of IL- 6, IL-1 $\beta$ and TNF- $\alpha$ and the phosphorylation of JAK2/STAT3 were downregulated in the SFN2.5, SFN5 and SFN10 groups compared with those in the $\mathrm{AD}$ group (Fig. 6B-E).

\section{Discussion}

AD is a chronic disease characterized by skin barrier dysfunction, IgE-mediated hypersensitivity and alterations in cell-mediated immune responses (23). Previous studies have suggested that oxidative stress, which can lead to skin barrier dysfunction, has a central role in the occurrence and development of $\mathrm{AD}$, and that this is related to poor prognosis of $\operatorname{AD~}(5,24)$. In the present study, sulforaphane treatment 

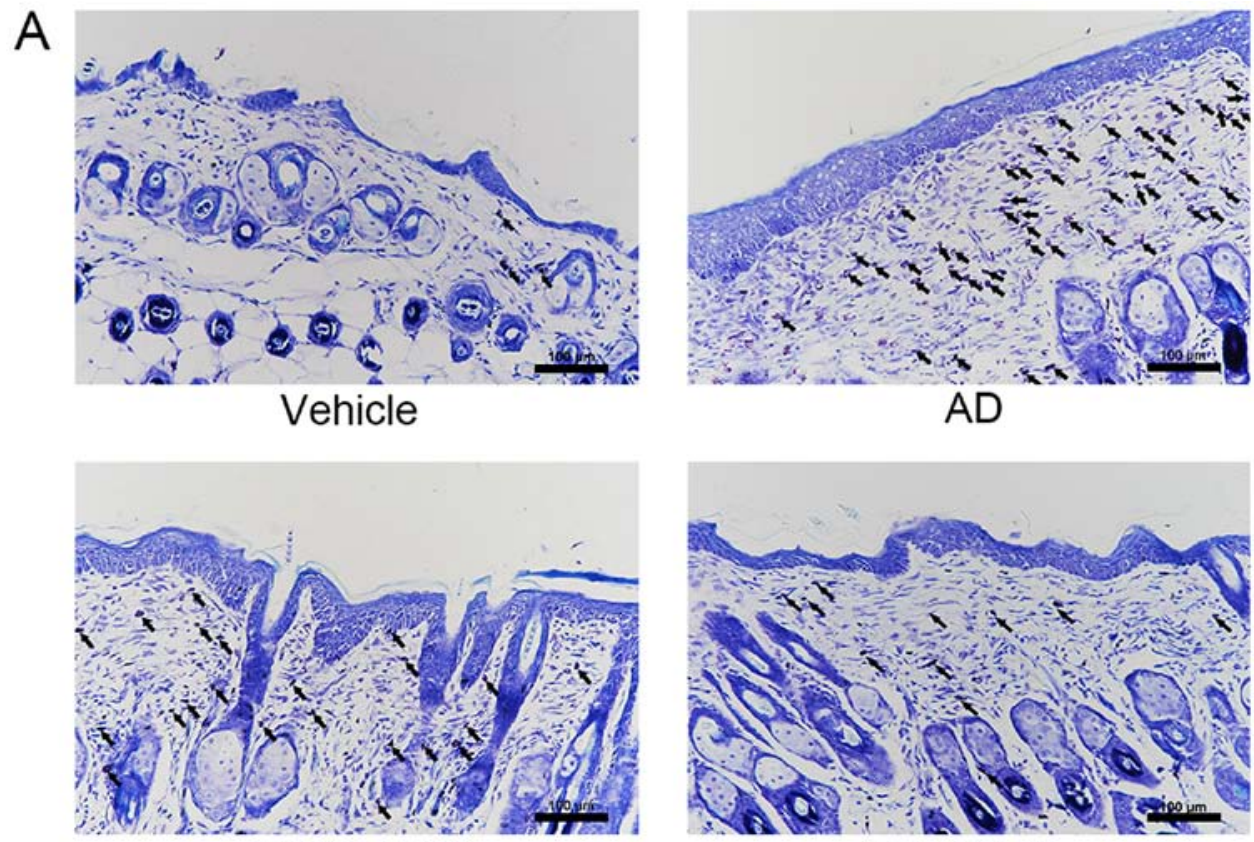

$A D+S F N 2.5$

AD+SFN5
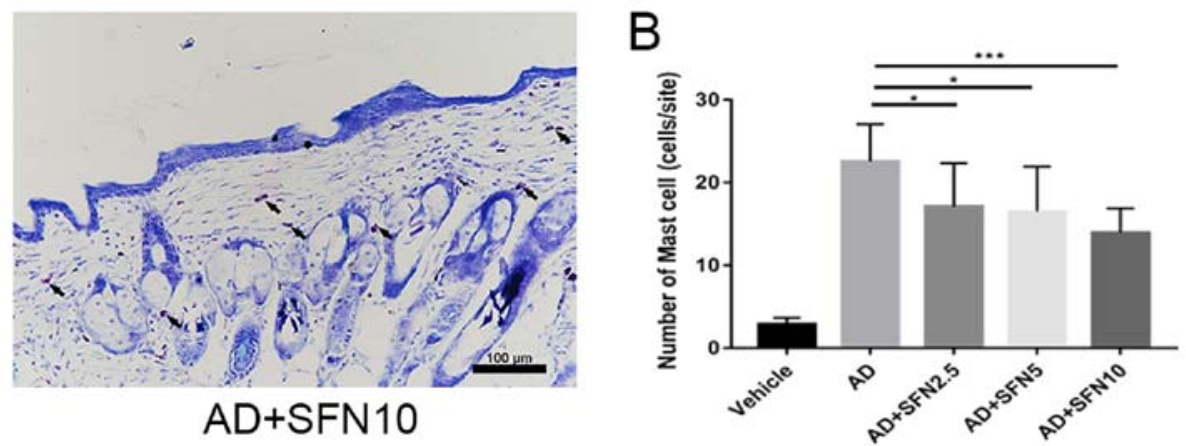

Figure 5. Effects of SFN on mast cell infiltration in atopic dermatitis mouse skin lesions and the expression of related molecules at the protein level (5 fields per animal). (A) Mast cells were presented in toluidine blue staining sections ( $\mathrm{x} 40$; scale bar, $100 \mu \mathrm{m}$ ). Black arrows indicate stained mast cells. (B) The average number of mast cells in five sites chosen randomly was counted at $x 400$ magnification. Results are expressed as the mean \pm SEM $(n=8)$. ${ }^{*} \mathrm{P}<0.05$ and ${ }^{* * *} \mathrm{P}<0.001$. One-way ANOVA analysis followed by Dunnett's post hoc test. DNCB, 2,4-dinitrochlorobenzene; SFN, sulforaphane; Vehicle, vehicle + PBS-treated group; $\mathrm{AD}, \mathrm{DNCB}+\mathrm{PBS}$-treated group; AD + SFN2.5, DNCB + SFN (2.5 mg/kg i.p.)-treated group; AD + SFN5, DNCB + SFN (5 mg/kg i.p.)-treated group; $\mathrm{AD}+\mathrm{SFN} 10, \mathrm{DNCB}+\mathrm{SFN}$ (10 mg/kg i.p.)-treated group.

alleviated the inflammation in $\mathrm{AD}$ mice, reduced the accumulation of eosinophils and mast cells in the epithelial tissue and reduced the expression levels of serum IgE. Furthermore, the expression levels of Nrf2 and HO-1 were upregulated in the epithelial tissue of AD mice following sulforaphane treatment.

Oxidative stress in turn can directly damage epithelial cells and induce eosinophil and mast cell accumulation, together with secretion of proinflammatory cytokines, which can cause dermal inflammation, which leads to itching and scratching. The dermal inflammation, and subsequent itching and scratching, in turn increase the release of proinflammatory cytokines; thus, these factors form a positive loop that can exaggerate AD illness (5). Sulforaphane, a natural isothiocyanate extracted from cruciferous vegetables such as broccoli, has a variety of potential abilities for the treatment of solid cancers, cardiovascular diseases, neurodegenerative diseases and diabetes (25). One of the major molecular mechanisms of action of sulforaphane is the activation of the Nrf2-Kelch-like $\mathrm{ECH}$-associated protein 1 pathway, which is involved in the response to damage caused by oxidant compounds (25). Nrf2, a transcription factor that regulates genes encoding antioxidant and detoxifying molecules, can ameliorate oxidative stress and inflammation in chronic kidney disease, cardiovascular disease and Alzheimer's disease in humans (26-28). Furthermore, $\mathrm{Nrf} 2$ is associated with epidermal barrier function to protect against oxidant damage (29). Choi et al (30) demonstrated that Platycodon grandiflorum root-derived saponins can improve AD-like lesions in mice by activating Nrf2/ARE. However, whether sulforaphane has similar therapeutic effects in an AD mouse model remains to be elucidated.

The present study used $2.5,5$ and $10 \mathrm{mg} / \mathrm{kg}$ sulforaphane to treatADmice. The concentrations of sulforaphane were in accordance with other studies on sulforaphane treatment $(18,19)$. As no studies on SFN in AD have been identified, the present study explored the appropriate therapeutic concentration of SFN in $\mathrm{AD}$, so that it can be used for further research. The comparison of studies on sulforaphane and clinically positive control drugs is also a matter for further study. Previous studies examined the use of SFN in UVB-induced skin treatment $(31,32)$ and demonstrated that SFN has the potential to treat skin disease 
A

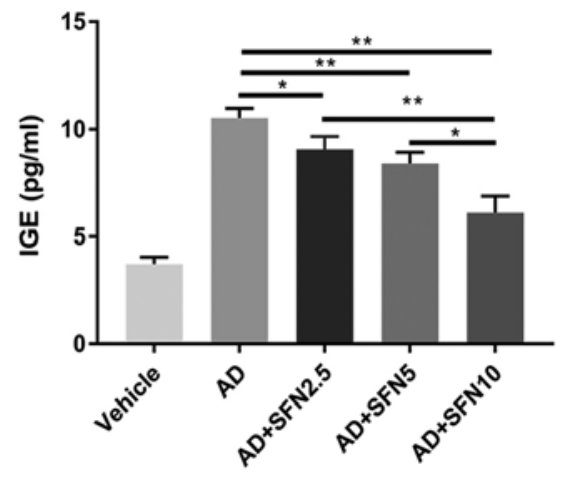

B

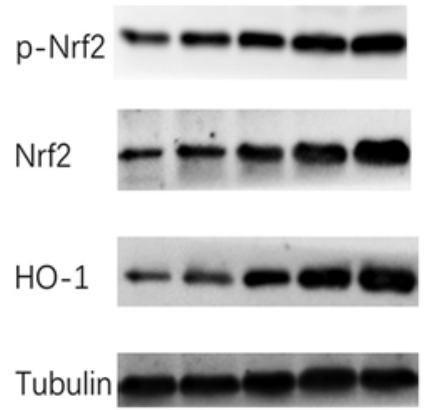

p-JAK1

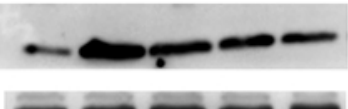

JAK1

p-STAT3

STAT3

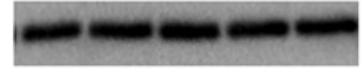

Tubulin

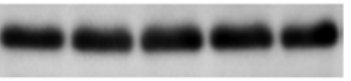

C

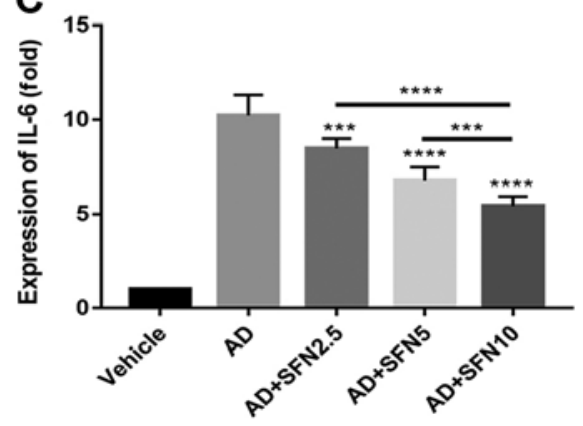

D

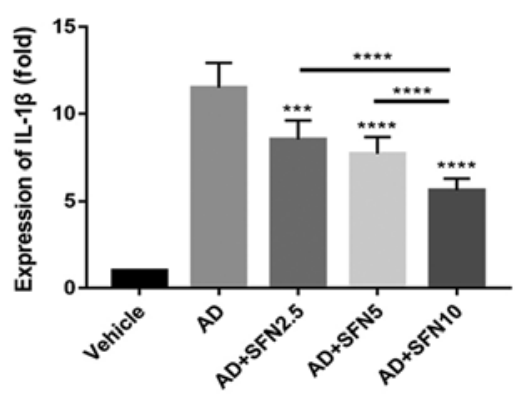

E

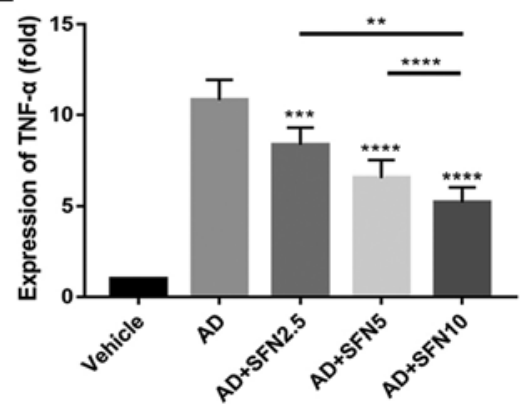

Figure 6. (A) Serum IgE levels were measured by ELISA. (B) Western blot revealed protein level changes in the expression of Nrf2, p-Nrf2, HO-1, p-JAK1, JAK1, p-STAT3 and STAT3. Tubulin was used as an internal reference. (C) Reverse transcription-quantitative PCR assay revealed mRNA level changes in the expression of (C) IL-6, (D) IL-1 $\beta$ and (E) TNF- $\alpha$. Results are expressed as the mean $\pm \mathrm{SEM}(\mathrm{n}=8)$. ${ }^{*} \mathrm{P}<0.05,{ }^{* * *} \mathrm{P}<0.01,{ }^{* * *} \mathrm{P}<0.001$ and ${ }^{* * * *} \mathrm{P}<0.0001$. One-way ANOVA analysis followed by Dunnett's post hoc test. DNCB, 2,4-dinitrochlorobenzene; SFN, sulforaphane; Vehicle, vehicle + PBS-treated group; AD, DNCB + PBS-treated group; AD + SFN2.5, DNCB + SFN (2.5 mg/kg i.p.)-treated group; AD+SFN5, DNCB + SFN (5 mg/kg i.p.)-treated group; $\mathrm{AD}+\mathrm{SFN} 10, \mathrm{DNCB}+\mathrm{SFN}(10 \mathrm{mg} / \mathrm{kg}$ i.p. $)$-treated group.

induced by immune-inflammation responses. The present study demonstrated that sulforaphane upregulated the expression levels of p-Nrf2 and Nrf2, and its downstream antioxidant molecule HO-1 in the epithelial cells of DNCB-induced AD mice, and downregulated the expression levels of IL-6, IL-1 $\beta$ and TNF- $\alpha$, and the phosphorylation of JAK2/STAT3. A previous study demonstrated that Nrf2 knockout mice require higher levels of inflammatory stimulation to initiate contact dermatitis compared with normal mice and that the presence of Nrf2 in keratinocytes limits inflammation (33). In contact dermatitis, Nrf2 improves the condition of patients and the activation of Nrf2 is required for the activation of the ARE reporter gene (34). Nrf2 is associated with epidermal barrier function for protection against oxidant damage (29). In UVA irradiation-related study, sulforaphane was considered as an anti-oxidative stress-associated agent to treat photoaging in $\mathrm{BALB} / \mathrm{c}$ mice and the activation of $\mathrm{Nrf} 2$ and reduction of MMP-1 induced by sulforaphane were observed (32). These studies suggested that $\mathrm{Nrf} 2$ has the ability to control epidermal inflammation and this conclusion is consistent with the experimental results of the present study. It is therefore concluded that sulforaphane exerted a therapeutic effect in the AD mouse model by the activation of the Nrf2/HO-1 axis. The present study also found that the phosphorylation of JAK2/STAT3 and the expression levels of IL-6, IL- $1 \beta$ and TNF- $\alpha$ were reduced in the SFN-treated group compared with the AD group. Welsch et al (35) reported that JAK/STAT signaling serves an important role in inflammatory skin diseases and that JAK/STAT inhibitors should presumably have many applications in dermatology. Jin et al (36) demonstrated that the JAK1/JAK2 inhibitor momelotinib inhibits the inflammatory response in DNCB-induced AD mice. Accordingly, the results of the present study indicated that SFN not only upregulated the Nrf2/HO-1 pathway, but also downregulated the JAK2/STAT3 pathway associated with inflammation. Abe and Tanaka (37) demonstrated that Nrf2 can attenuate the expression of IL- 6 and IL-1 $\beta$, which decreased the macrophage inflammatory response. The study of Chu et al (38), demonstrated that the expression levels of IL- 6, IL- $1 \beta$ and TNF- $\alpha$ are significantly inhibited by SFN in a rheumatoid arthritis model. These results demonstrate that $\mathrm{Nrf} 2$ can regulate the downstream cytokines, including IL- 6 , IL- $1 \beta$ and TNF- $\alpha$, to influence the progression of inflammation in AD.

However, there are some limitations to the present study. In a previous study, Roy et al (39) demonstrated that the expression of $\mathrm{NF}-\kappa \mathrm{B}$ and mitogen-activated protein kinases (MAPKs), as inflammatory mediators, are associated with the progression of AD. In addition, the decline of MAPKs and $\mathrm{NF}-\kappa \mathrm{B}$ alleviates AD symptoms (40). Pastore et al (41) reported that activation of c-JUN/c-FOS pathway promotes inflammation in AD. However, there are no studies, to the best of our knowledge, which report that the expression of c-JUN/c-FOS, $\mathrm{NF}-\kappa \mathrm{B}$ and MAPKs are associated with SFN treatment in AD. Further studies are required to verify the inflammation mediator function in the SFN treatment of AD and that was a limitation of the present study. The results demonstrated a new approach to treat dermatitis by activating skin protective molecules that can enhance the skin barrier and the role of 
this mechanism in specific dermatitis needs to be identified in future experiments.

In patients with $\mathrm{AD}$, mast cell numbers are increased in skin lesions (40). Although the function and status of mast cells in $\mathrm{AD}$ is not clear, there is a potential mechanism by which mast cells contribute to AD progression, whereby mast cells release cytokines such as IL-17 and IL-22 that induce epithelial inflammation and allergic response (42). In addition, mast cells can intensify scratching damage by releasing pruritogenic substances (43). This further disrupts the skin barrier and exacerbates the disease. In previous studies, the Nrf2-HO-1 pathway was found to mediate anti-allergic actions in rodent mast cells and HO-1 was shown to control inflammation by stabilizing mast cells (43-45). In the present study, AD mice that underwent sulforaphane treatment demonstrated a decline in mast cells in skin in the SFN2.5, SFN5 and SFN10 groups compared with the AD group. Combined with the results of previous studies, these results indicate that sulforaphane can control inflammation by inhibiting mast cell infiltration through the Nrf2-HO-1 pathway.

A consensus has been reached that elevated serum IgE levels in patients with AD are directly associated with poor prognosis and more severe development of AD (11). The anti-IgE antibody omalizumab was used to treat severe AD along with extracorporeal immunoadsorption and the combination therapy resulted in an improvement in $\mathrm{AD}$ during the treatment period (46). In a recent study, 6-shogaol, an active compound present in ginger, was found to activate the reactive oxygen species (ROS)/MAPKs/Nrf2 anti-inflammatory pathway to alleviate AD-like skin lesions by inhibiting the development of DNCB-induced AD-like skin lesions and scratching behavior, and reducing the expression of $\operatorname{IgE}$ and ROS generation (47). The present study identified that serum IgE levels in the AD group were $>2$ times higher compared with the vehicle group and that there was a significant decrease in $\operatorname{IgE}$ levels in the SFN2.5, SFN5 and SFN10 groups compared with the AD group. It was also identified that as the drug concentration increased gradually, the serum IgE levels in the SFN2.5, SFN5 and SFN10 groups decreased compared with the AD group. Eosinophil infiltration is characteristic of patients with AD and it is associated with disease severity (48). Eosinophils are involved in T helper 2 (Th2)-immune response in AD and Th2/Th22-dominant allergic responses, which are considered as the major molecular mechanism in AD. In addition, Th2 cytokines induce oxidative stress and severe inflammatory response, and lead to the aggravation of AD (49). Infiltration of eosinophils amplifies Th2-immune responses and Th2 cells begin secreting IL-4, IL-5 and IL-13, which augments skin inflammation $(43,50)$. A recent study (49) reported notable findings about AD; dupilumab, an anti-IL-4-receptor- $\alpha$ monoclonal antibody, can block signaling of IL-4 and IL-13, which are type $2 / \mathrm{Th} 2$ cytokines implicated in numerous allergic diseases, including AD. In this 1-year, randomized, double-blinded, placebo-controlled, phase 3 trial, signs and symptoms of patients with $\mathrm{AD}$ were alleviated by dupilumab with acceptable safety. It can be concluded that the reduction of type $2 / \mathrm{Th} 2$ will be a key factor for the treatment of $\mathrm{AD}$ and that the accumulation of eosinophils in skin could be considered as a therapeutic effect indicator, as the accumulation of eosinophils is suppressed by sulforaphane treatment in AD mice. In the present study, AD mice that underwent sulforaphane treatment (SFN2.5, SFN5 and SFN10 groups) also demonstrated a decrease in eosinophil infiltration in skin compared with the AD group. The results of the present study demonstrated that sulforaphane can downregulate the level of IgE in DNCB-induced AD mice, alleviate the edema and itching, and reduce the infiltration of eosinophils and mast cells; thus, it could be considered as a potential agent to treat $\mathrm{AD}$.

In conclusion, the results of the present study demonstrated that sulforaphane alleviated AD symptoms in DNCB-induced AD mice, potentially through the activation of the Nrf2/HO-1 pathway and the suppression of JAK1/STAT3 signaling, and that sulforaphane may be a potential therapeutic option for treating patients with AD.

\section{Acknowledgements}

Not applicable.

\section{Funding}

The present study was supported by Scientific Research Key Project of Educational Department in Liaoning Province of China in 2017 (grant no. 2017225026) and Science and Technology Project of Shenyang (grant no. 17-230-9-25). The funders had no role in study design, data collection and analysis, decision to publish, or preparation of the manuscript.

\section{Availability of data and materials}

All data generated or analyzed during this study are included in this published article.

\section{Authors' contributions}

WW induced AD-mice by DNCB, executed sulforaphane administration performed $\mathrm{H} \& \mathrm{E}$ and toluidine blue staining for measurement of epidermal thickness, eosinophils and mast cells of each group, and respectively and analyzed these data. GP evaluated the severity of dermatitis, scratching behavior and ear thickness of each group and analyzed these data. FY measured serum IgE expression of each group by Elisa and analyzed these data. YZ measured mRNA level of IL-6, IL-1 $\beta$ and TNF- $\alpha$ of each group and analyzed these data. ZM performed western blot analysis for $\mathrm{Nrf} 2 / \mathrm{HO}-1$ and JAK1/STAT3 pathway in each group and analyzed these data. $\mathrm{XH}$ was a major contributor to the study design and data interpretation. All authors read and approved the final manuscript.

\section{Ethics approval and consent to participate}

The study procedures were approved by the ethics committee of Shengjing Hospital of China Medical University.

\section{Patient consent for publication}

Not applicable.

\section{Competing interests}

The authors declare that they have no competing interests. 


\section{References}

1. Bieber T: Atopic dermatitis. N Engl J Med 358: 1483-1494, 2008

2. Weidinger $S$ and Novak N: Atopic dermatitis. Lancet 387: 1109-1122, 2016.

3. Werfel T, Schwerk N, Hansen G and Kapp A: The diagnosis and graded therapy of atopic dermatitis. Dtsch Arztebl Int 111: 509-520, 2014

4. Gęgotek A and Skrzydlewska E: The role of transcription factor Nrf2 in skin cells metabolism. Arch Dermatol Res 307: 385-396, 2015.

5. Ji H and Li XK: Oxidative stress in atopic dermatitis. Oxid Med Cell Longev 2016: 2721469, 2016.

6. Rojo de la Vega M, Krajisnik A, Zhang DD and Wondrak GT: Targeting NRF2 for improved skin barrier function and photoprotection: Focus on the achiote-derived apocarotenoid bixin. Nutrients 9: E1371, 2017.

7. Gruber F, Ornelas CM, Karner S, Narzt MS, Nagelreiter IM, Gschwandtner M, Bochkov V and Tschachler E: Nrf2 deficiency causes lipid oxidation, inflammation and matrix-protease expression in DHA supplemented and UVA irradiated skin fibroblasts. Free Radic Biol Med 88: 439-451, 2015.

8. Guerrero-Beltrán CE, Calderón-Oliver M, Pedraza-Chaverri J and Chirino YI: Protective effect of sulforaphane against oxidative stress: Recent advances. Exp Toxicol Pathol 64: 503-508, 2012

9. Mathew ST, Bergström P and Hammarsten O: Repeated Nrf2 stimulation using sulforaphane protects fibroblasts from ionizing radiation. Toxicol Appl Pharmacol 276: 188-194, 2014.

10. Kumar KJ, Yang HL, Tsai YC, Hung PC, Chang SH, Lo HW, Shen PC, Chen SC, Wang HM, Wang SY, et al: Lucidone protects human skin keratinocytes against free radical-induced oxidative damage and inflammation through the up-regulation of $\mathrm{HO}-1 / \mathrm{Nrf} 2$ antioxidant genes and down-regulation of NF- $\mathrm{KB}$ signaling pathway. Food Chem Toxicol 59: 55-66, 2013.

11. Furue M, Chiba T, Tsuji G, Ulzii D, Kido-Nakahara M, Nakahara T and Kadono T: Atopic dermatitis: Immune deviation, barrier dysfunction, IgE autoreactivity and new therapies. Allergol Int 66: 398-403, 2017.

12. Kiiski V, Karlsson O, Remitz A and Reitamo S: High serum total IgE predicts poor long-term outcome in atopic dermatitis. Acta Derm Venereol 95: 943-947, 2015.

13. Lucae S, Schmid-Grendelmeier P, Wüthrich B, Kraft D, Valenta R and Linhart B: IgE responses to exogenous and endogenous allergens in atopic dermatitis patients under long-term systemic cyclosporine A treatment. Allergy 71: 115-118, 2016.

14. Altrichter S, Kriehuber E, Moser J, Valenta R, Kopp T and Stingl G: Serum IgE autoantibodies target keratinocytes in patients with atopic dermatitis. J Invest Dermatol 128: 2232-2239, 2008.

15. Liu FT, Goodarzi H and Chen HY: IgE, mast cells, and eosinophils in atopic dermatitis. Clin Rev Allergy Immunol 41: 298-310, 2011.

16. Elovic A, Wong DT, Weller PF, Matossian K and Galli SJ: Expression of transforming growth factors-alpha and beta 1 messenger RNA and product by eosinophils in nasal polyps. J Allergy Clin Immunol 93: 864-869, 1994.

17. Kato A, Chustz RT, Ogasawara T, Kulka M, Saito H, Schleimer RP and Matsumoto K: Dexamethasone and FK506 inhibit expression of distinct subsets of chemokines in human mast cells. J Immunol 182: 7233-7243, 2009.

18. Zhang JC, Yao W, Dong C, Yang C, Ren Q, Ma M, Han M, Wu J, Ushida Y, Suganuma H and Hashimoto K: Prophylactic effects of sulforaphane on depression-like behavior and dendritic changes in mice after inflammation. J Nutr Biochem 39: 134-144, 2017.

19. Yan B, Ma Z, Shi S, Hu Y, Ma T, Rong G and Yang J: Sulforaphane prevents bleomycin-induced pulmonary fibrosis in mice by inhibiting oxidative stress via nuclear factor erythroid 2-related factor-2 activation. Mol Med Rep 15: 4005-4014, 2017.

20. O'Connor JC, Lawson MA, André C, Moreau M, Lestage J, CastanonN,KelleyKWandDantzerR:Lipopolysaccharide-induced depressive-like behavior is mediated by indoleamine 2,3-dioxygenase activation in mice. Mol Psychiatry 14: 511-522, 2009.

21. Peng G, Mu Z, Cui L, Liu P, Wang Y, Wu W and Han X: Anti-IL-33 antibody has a therapeutic effect in an atopic dermatitis murine model induced by 2, 4-dinitrochlorobenzene. Inflammation 41: 154-163, 2018

22. Livak KJ and Schmittgen TD: Analysis of relative gene expression data using real-time quantitative PCR and the 2(-Delta Delta C(T)) method. Methods 25: 402-408, 2001.
23. David Boothe W, Tarbox JA and Tarbox MB: Atopic dermatitis: Pathophysiology. Adv Exp Med Biol 1027: 21-37, 2017.

24. Briganti S and Picardo M: Antioxidant activity, lipid peroxidation and skin diseases. What's new. J Eur Acad Dermatol Venereol 17: 663-669, 2010.

25. Yang L, Palliyaguru DL and Kensler TW: Frugal chemoprevention: Targeting Nrf2 with foods rich in sulforaphane. Semin Oncol 43: 146-153, 2016.

26. Ruiz S, Pergola PE, Zager RA and Vaziri ND: Targeting the transcription factor Nrf2 to ameliorate oxidative stress and inflammation in chronic kidney disease. Kidney Int 83: 1029-1041, 2013.

27. Lawal AO: Air particulate matter induced oxidative stress and inflammation in cardiovascular disease and atherosclerosis: The role of Nrf2 and AhR-mediated pathways. Toxicol Lett 270 88-95, 2017

28. Prasad KN: Simultaneous activation of Nrf2 and elevation of antioxidant compounds for reducing oxidative stress and chronic inflammation in human Alzheimer's disease. Mech Ageing Dev 153: 41-47, 2016.

29. Schäfer M, Farwanah H, Willrodt AH, Huebner AJ, Sandhoff K, Roop D, Hohl D, Bloch W and Werner S: Nrf2 links epidermal barrier function with antioxidant defense. EMBO Mol Med 4: 364-379, 2012.

30. Choi JH, Jin SW, Han EH, Park BH, Kim HG, Khanal T, Hwang YP, Do MT, Lee HS, Chung YC, et al: Platycodon grandiflorum root-derived saponins attenuate atopic dermatitis-like skin lesions via suppression of NF- $\mathrm{KB}$ and STAT1 and activation of Nrf2/ARE-mediated heme oxygenase-1. Phytomedicine 21: $1053-1061,2014$.

31. Saw CL, Huang MT, Liu Y, Khor TO, Conney AH and Kong AN: Impact of Nrf2 on UVB-induced skin inflammation/ photoprotection and photoprotective effect of sulforaphane. Mol Carcinog 50: 479-486, 2011.

32. Chaiprasongsuk A, Lohakul J, Soontrapa K, Sampattavanich S, Akarasereenont $\mathrm{P}$ and Panich $\mathrm{U}$ : Activation of Nrf2 reduces UVA-mediated MMP-1 upregulation via MAPK/AP-1 signaling cascades: The photoprotective effects of sulforaphane and hispidulin. J Pharmacol Exp Ther 360: 388-398, 2017.

33. Migdal C, Botton J, El Ali Z, Azoury ME, Guldemann J, Giménez-Arnau E, Lepoittevin JP, Kerdine-Römer S and Pallardy M: Reactivity of chemical sensitizers toward amino acids in cellulo plays a role in the activation of the Nrf2-ARE pathway in human monocyte dendritic cells and the THP-1 cell line. Toxicol Sci 133: 259-274, 2013.

34. Emter R, van der Veen JW, Adamson G, Ezendam J, van Loveren $\mathrm{H}$ and Natsch A: Gene expression changes induced by skin sensitizers in the KeratinoSens ${ }^{\mathrm{TM}}$ cell line: Discriminating Nrf2-dependent and Nrf2-independent events. Toxicol In Vitro 27: 2225-2232, 2013.

35. Welsch K, Holstein J, Laurence A and Ghoreschi K: Targeting JAK/STAT signalling in inflammatory skin diseases with small molecule inhibitors. Eur J Immunol 47: 1096-1107, 2017.

36. Jin W, Huang W, Chen L, Jin M, Wang Q, Gao Z and Jin Z: Topical application of JAK1/JAK2 inhibitor momelotinib exhibits significant anti-inflammatory responses in DNCB-induced atopic dermatitis model mice. Int J Mol Sci 19: E3973, 2018.

37. Abe $\mathrm{Y}$ and Tanaka N: The Hedgehog signaling networks in lung cancer: The mechanisms and roles in tumor progression and implications for cancer therapy. Biomed Res Int 2016: 7969286, 2016.

38. Chu J, Wang X, Bi H, Li L, Ren M and Wang J: Dihydromyricetin relieves rheumatoid arthritis symptoms and suppresses expression of pro-inflammatory cytokines via the activation of $\mathrm{Nrf} 2$ pathway in rheumatoid arthritis model. Int Immunopharmacol 59: 174-180, 2018.

39. Roy R, Dagher A, Butterfield C and Moses MA: ADAM12 is a novel regulator of tumor angiogenesis via STAT3 signaling. Mol Cancer Res 15: 1608-1622, 2017.

40. Choi JK, Jang YH, Lee S, Lee SR, Choi YA, Jin M, Choi JH, Park JH, Park PH, Choi H, et al: Chrysin attenuates atopic dermatitis by suppressing inflammation of keratinocytes. Food Chem Toxicol 110: 142-150, 2017.

41. Pastore S, Giustizieri ML, Mascia F, Giannetti A, Kaushansky K and Girolomoni G: Dysregulated activation of activator protein 1 in keratinocytes of atopic dermatitis patients with enhanced expression of granulocyte/macrophage-colony stimulating factor. J Invest Dermatol 115: 1134-1143, 2000.

42. Mashiko S, Bouguermouh S, Rubio M, Baba N, Bissonnette R and Sarfati M: Human mast cells are major IL-22 producers in patients with psoriasis and atopic dermatitis. J Allergy Clin Immunol 136: 351-359.e1, 2015. 
43. Mu Z,Zhao Y, Liu X, Chang C and Zhang J: Molecular biology of atopic dermatitis. Clin Rev Allergy Immunol 47: 193-218, 2014.

44. Matsushima M, Takagi K, Ogawa M, Hirose E, Ota Y Abe F, Baba K, Hasegawa T, Hasegawa Y and Kawabe T: Heme oxygenase-1 mediates the anti-allergic actions of quercetin in rodent mast cells. Inflammation Res 58: 705-715, 2009.

45. Ma YY, Yang MQ, Wang CF, Ding J and Li JY: Inhibiting mast cell degranulation by $\mathrm{HO}-1$ affects dendritic cell maturation in vitro. Inflamm Res 63: 527-537, 2014

46. Zink A, Gensbaur A, Zirbs M, Seifert F, Suarez IL, Mourantchanian V, Weidinger S, Mempel M, Ring J and Ollert M: Targeting IgE in severe atopic dermatitis with a combination of immunoadsorption and omalizumab. Acta Derm Venereol 96: 72-76, 2016.

47. Park G, Oh DS, Lee MG, Lee CE and Kim YU: 6-Shogaol, an active compound of ginger, alleviates allergic dermatitis-like skin lesions via cytokine inhibition by activating the Nrf2 pathway. Toxicol Appl Pharmacol 310: 51-59, 2016.
48. Kiehl P, Falkenberg K, Vogelbruch M and Kapp A: Tissue eosinophilia in acute and chronic atopic dermatitis: A morphometric approach using quantitative image analysis of immunostaining. Br J Dermatol 145: 720-729, 2001.

49. Ajith Y, Dimri U, Gopalakrishnan A, Madhesh E, Jhambh R, Joshi V and Devi G: Th1/Th2 immune responses and oxidative stress in caprine flea allergy dermatitis. Parasite Immunol 39, 2017.

50. Werfel T, Allam JP, Biedermann T, Eyerich K, Gilles S, Guttman-Yassky E, Hoetzenecker W, Knol E, Simon HU, Wollenberg A, et al: Cellular and molecular immunologic mechanisms in patients with atopic dermatitis. J Allergy Clin Immunol 138: 336-349, 2016

(i) (9) This work is licensed under a Creative Commons Attribution-NonCommercial-NoDerivatives 4.0 International (CC BY-NC-ND 4.0) License. 